\title{
Programming And Load Balancing For The Development Of The Multifamily Building - Lima, Peru 2020
}

\author{
Robert Calvo1 $^{\text {a }}$, Doris Esenarro ${ }^{\mathrm{b}}$, Franz Hernandez $^{\mathrm{c}}$, Lorena Vela ${ }^{\mathrm{d}}$, Raul Mendez \\ a,c,d5EUPG Graduate School Federico Villarreal University - UNFV \\ ${ }^{b, e}$ Federico Villalrreal National University, Lima-Peru.

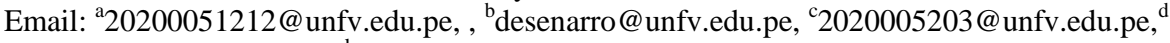 \\ 2020005429@unfv.edu.pe, ${ }^{b}$ desenarro@unfv.edu.pe, ${ }^{e}$ rmendez@unfv.edu.pe
}

Article History: Received: 10 November 2020; Revised 12 January 2021 Accepted: 27 January 2021; Published online: 5 April 2021

\begin{abstract}
The objective of this research is to show the production management in the construction of a multifamily building in Lima - Peru, it is worth mentioning that the main problem is that the companies do not adequately plan the optimal use of resources, the plan of attack of work, the sequence of activities through a correct design of production batch and sizing of crews, which are decisive during the execution of the work. The methodology used in this context is load and crew balancing. This allows us to manage resources and work scheduling, resulting in $100 \%$ efficiency. In conclusion, we can say that companies must maintain a correct control of their resources in order to achieve their schedules.
\end{abstract}

Keywords: y

\section{Introduction}

The construction sector has continuously been syndicated to imperfect performance. In general, the impression is that construction is a sector of low productivity and dubious quality given the lack of specialization of workers and professionals in the sector, many of the problems mentioned above are generated due to the lack of planning of the works, since the problems are solved as they appear. Although it is true that there are inconveniences that appear unexpectedly, many of the obstacles to the normal production of a task are predictable. Some professionals believe that the results of their design and construction management work had been completed within budget. However, these data are followed by those who completed their projects in the construction phase more than $3 \%$ over budget in the design phase, under $3 \%$. In terms of time, this was not good in the construction phase, with most finishing less than 15 days behind schedule. [1].

Nowadays, construction companies not only offer lower cost works, but also quality, safety and time. Therefore, they seek to optimize their work times, generating efficiency and profit for the company. This uncontrolled number of projects has a main "defect" that comes to light and is incredibly costly: the waste or losses that are generated in the construction stage of these projects. [2]. These companies have been implementing the Lean Construction philosophy, achieving favorable results in terms of profit margins, productivity, labor efficiency and safety [3].

The traditional construction planning is based on making the work plan with scheduled dates, visualizing the duration of the items, as well as the total time of execution of the work. However, the optimal use of resources, the work attack plan, the sequence of activities through a correct design of production batch and sizing of crews, which are decisive during the execution of the work, are not adequately planned [4], which is why this article aims to show how production could be managed in the construction of a multifamily building in Lima - Peru. The essence of the system is to work to increase the reliability of the planning. The ultimate planner is the one who finally defines what will be done and who will do the work. The role of ultimate planner can be held by foremen, construction managers, supervisors, subcontractors, site managers and others. [5]

\section{Method}

\section{Study area}

The study was conducted in Peru, in the city of metropolitan Lima. (Figure 1). 

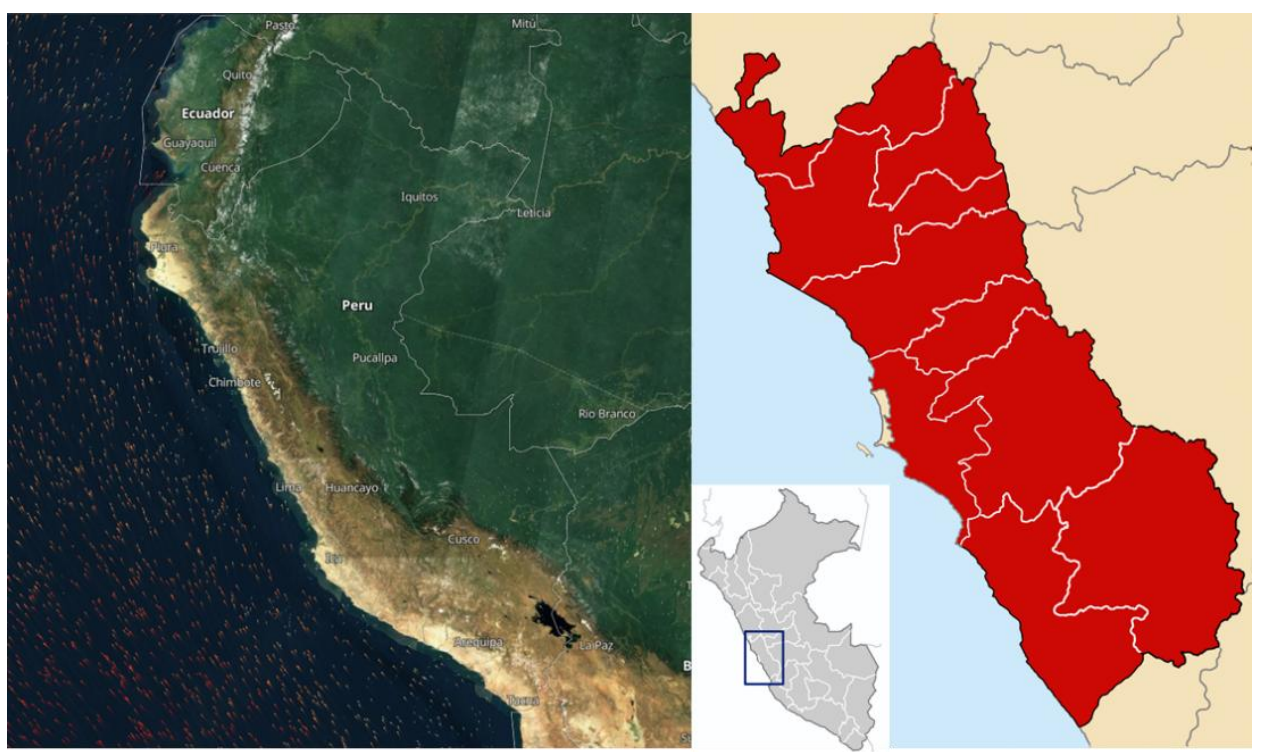

Figure 1. Localización de área de estudio.

Figure 1 shows the location of the study area, framed in the department of Lima

\section{Type of research}

The quantitative research will analyze the facts prior to and during the execution of the multifamily buildings, for this the experience must be taken into account and thus analyze correctly and concisely in our study. Likewise, the personnel resources to be used will be analyzed. From the information, the results will be obtained to make the corresponding decisions. [6

\section{Procedure}

Table $\mathbf{N}^{\circ} \mathbf{1}$. Metrication per unit of element

METERING PER UNIT OF ELEMENT

\begin{tabular}{ccccc}
\hline & & & ENCOUNTERED & \\
ELEMENT & NAME & STEEL $(\mathrm{kg})$ & CONCRETE $(\mathrm{m} 3)$ & 10.00 \\
\hline \hline Verticals & C1 & 1000.00 & 40.00 & 5.00 \\
Verticals & C2 & 500.00 & 20.00 & 7.50 \\
Verticals & C3 & 750.00 & 30.00 & 7.00 \\
Horizontals & P1 & 1250.00 & 30.00 & 9.00 \\
Horizontals & P2 & 1500.00 & 40.00 & 5.00 \\
Horizontals & P3 & 1000.00 & 20.00 & \\
\hline
\end{tabular}

Table $\mathbf{N}^{\circ} \mathbf{1}$ shows the corresponding metrics for the different elements used in the project. 


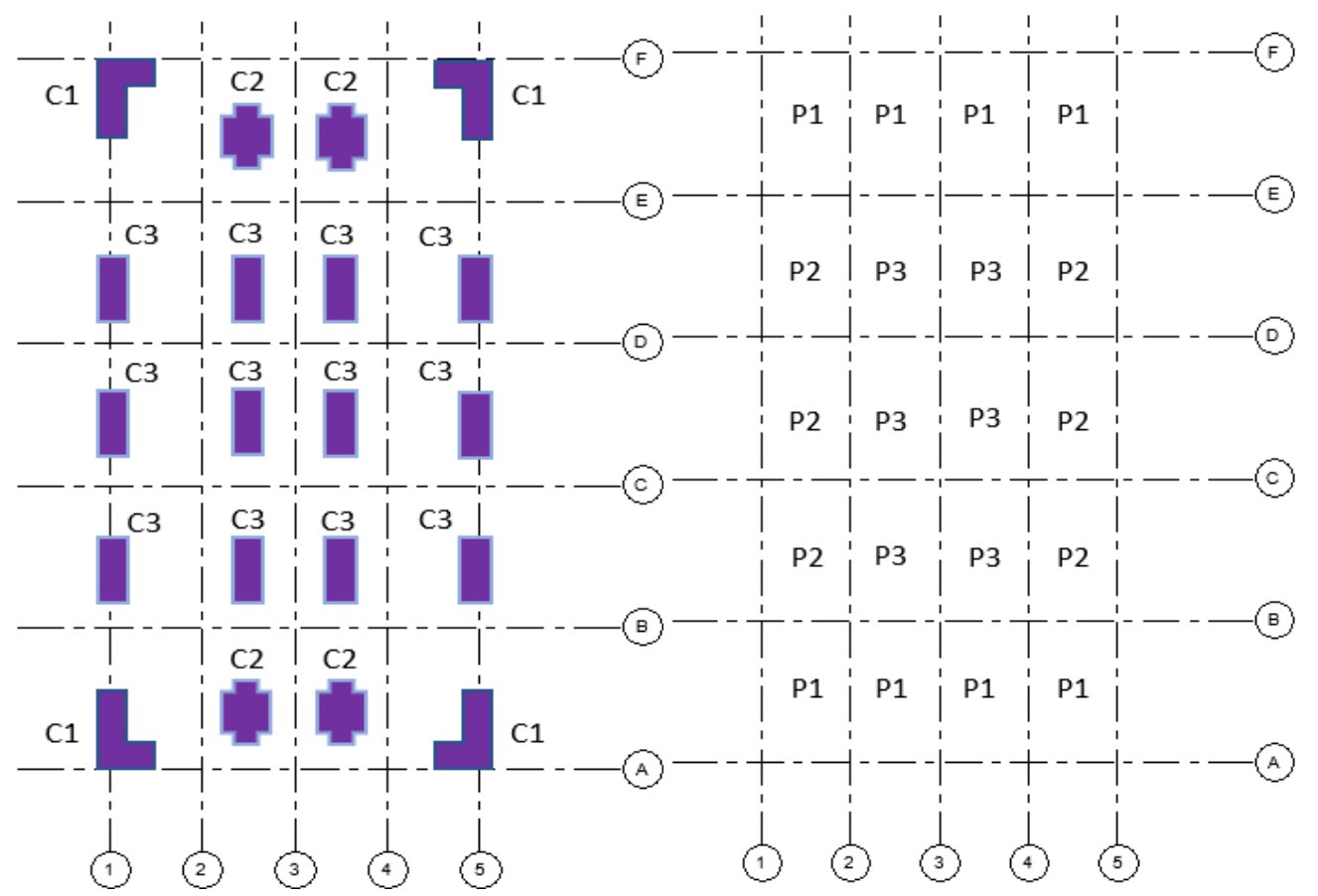

Figura 2. Verticals Plan

Figure 3. Horizontals Plan

For practical purposes, only the vertical and horizontal items will be taken as shown in Figure 2 and 3

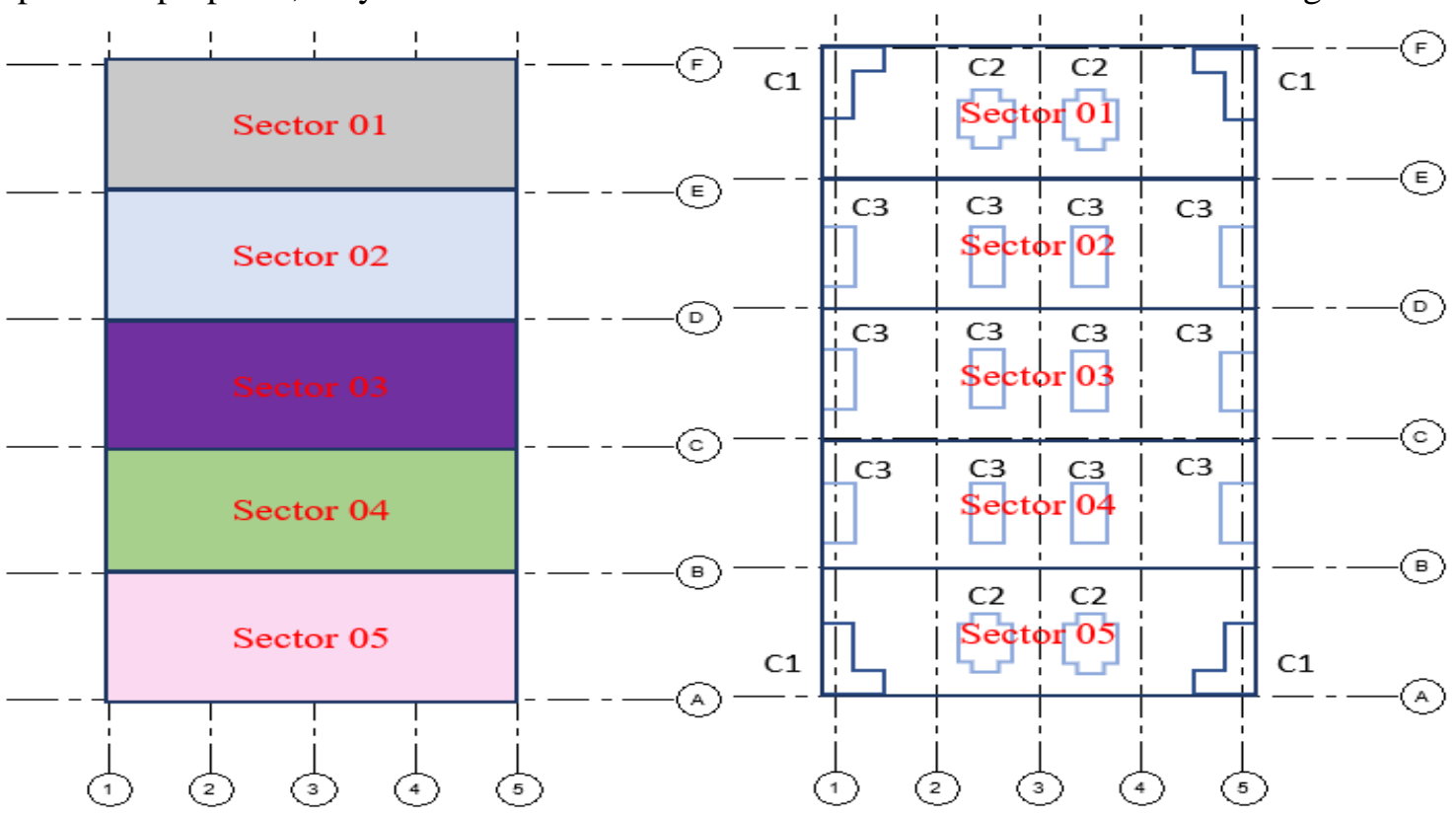

Figure 4. Vertical sectorization plan

Figure 5.Horizontal sectorization pla In Figure 4 and 5 shows the metrics, we proceed to sectorize the work area which consists of dividing a task or activity of the work in sectors, a small part of the total task should be included and in turn should include an approximately equal metric trying to balance the loads as shown. [7]

Sectoring in a correct way allows us to obtain greater efficiency, improve control and maximize production capacity.

Table $\mathbf{N}^{\circ}$ 2. Vertical metering

\begin{tabular}{|c|c|c|c|c|c|c|c|}
\hline \multicolumn{8}{|c|}{ METRADO DE VERTICALES } \\
\hline \multirow{2}{*}{$\begin{array}{l}\text { SECT } \\
\text { OR }\end{array}$} & \multirow{2}{*}{$\begin{array}{l}\text { ELEM } \\
\text { ENT }\end{array}$} & \multicolumn{3}{|c|}{ NAME } & \multirow{2}{*}{$\begin{array}{c}\text { TOTAL } \\
\text { METER STEEL } \\
(\mathrm{Kg}) \\
\end{array}$} & \multirow{2}{*}{$\begin{array}{c}\text { TOTAL } \\
\text { FORMWORK } \\
\text { LENGTH (m2) } \\
\end{array}$} & \multirow{2}{*}{$\begin{array}{c}\text { TOTAL } \\
\text { CONCRETE } \\
\text { THICKNESS (m3) } \\
\end{array}$} \\
\hline & & $\mathrm{C}$ & $\mathrm{C}$ & $\mathrm{C}$ & & & \\
\hline
\end{tabular}




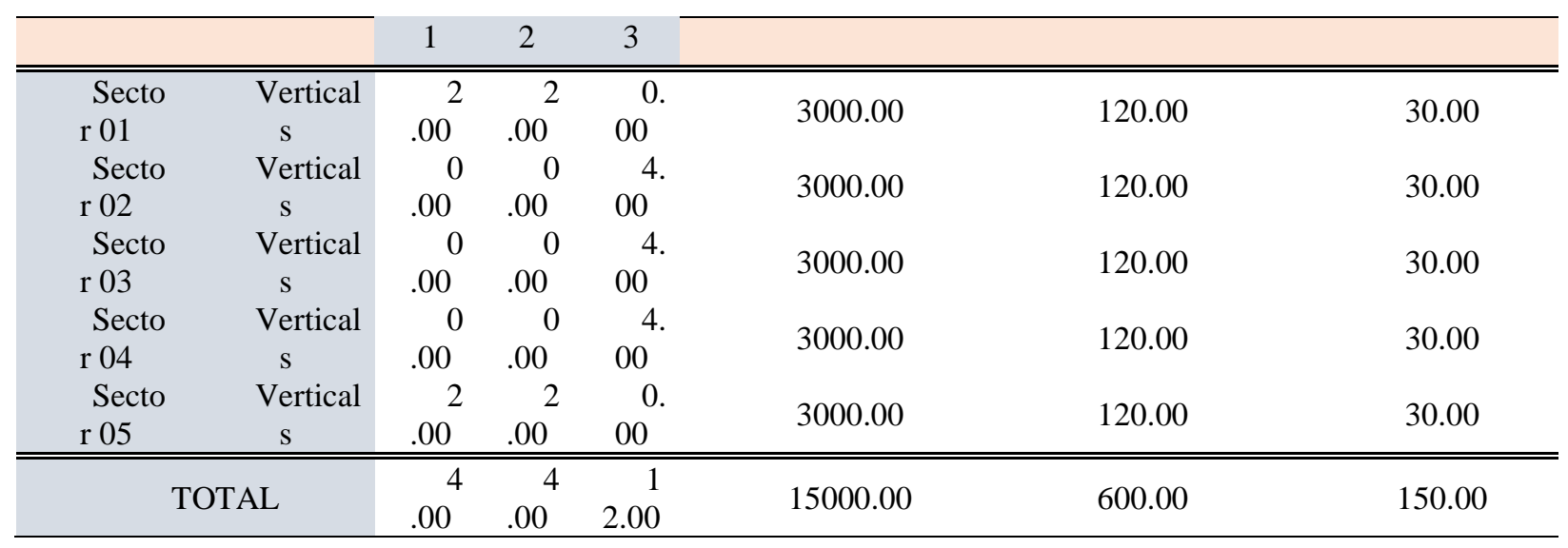

Table $\mathrm{N}^{\circ} 2$ below shows the vertical element metrics according to the sectorization carried out.

Table $\mathbf{N}^{\circ}$ 3. Horizontal metering

\begin{tabular}{|c|c|c|c|c|c|c|c|}
\hline \multicolumn{8}{|c|}{ METERING OF HORIZONTALS } \\
\hline \multirow{2}{*}{$\begin{array}{l}\text { SECT } \\
\text { OR }\end{array}$} & \multirow{2}{*}{$\begin{array}{l}\text { ELEM } \\
\text { ENT }\end{array}$} & \multicolumn{3}{|c|}{ NAME } & \multirow{2}{*}{$\begin{array}{c}\text { TOTAL } \\
\text { METER STEEL } \\
(\mathrm{Kg})\end{array}$} & \multirow{2}{*}{$\begin{array}{c}\text { TOTAL } \\
\text { FORMWORK } \\
\text { LENGTH }(\mathrm{m} 2)\end{array}$} & \multirow{2}{*}{$\begin{array}{c}\text { TOTAL } \\
\text { CONCRETE } \\
\text { THICKNESS }(\mathrm{m} 3)\end{array}$} \\
\hline & & ${ }_{1}^{P}$ & $2^{P}$ & $3^{P}$ & & & \\
\hline $\begin{array}{l}\text { Secto } \\
\text { r } 01\end{array}$ & $\begin{array}{l}\text { Horizon } \\
\text { tals }\end{array}$ & $\begin{array}{l}{ }^{4} \\
.00\end{array}$ & $\begin{array}{r}0 \\
.00\end{array}$ & $\begin{array}{r}0 \\
.00\end{array}$ & 5000.00 & 120.00 & 28.00 \\
\hline $\begin{array}{l}\text { Secto } \\
\text { r } 02\end{array}$ & $\begin{array}{l}\text { Horizon } \\
\text { tals }\end{array}$ & $\begin{array}{c}0 \\
.00\end{array}$ & $\begin{array}{r}2 \\
.00\end{array}$ & $\begin{array}{r}2 \\
.00\end{array}$ & 5000.00 & 120.00 & 28.00 \\
\hline $\begin{array}{l}\text { Secto } \\
\text { r } 03\end{array}$ & $\begin{array}{l}\text { Horizon } \\
\text { tals }\end{array}$ & .00 & $\begin{array}{r}2 \\
.00\end{array}$ & $\begin{array}{r}2 \\
.00\end{array}$ & 5000.00 & 120.00 & 28.00 \\
\hline $\begin{array}{l}\text { Secto } \\
\text { r } 04\end{array}$ & $\begin{array}{l}\text { Horizon } \\
\text { tals }\end{array}$ & $\begin{array}{c}0 \\
.00\end{array}$ & $\begin{array}{r}2 \\
.00\end{array}$ & $\begin{array}{r}2 \\
.00\end{array}$ & 5000.00 & 120.00 & 28.00 \\
\hline $\begin{array}{l}\text { Secto } \\
\text { r } 05\end{array}$ & $\begin{array}{l}\text { Horizon } \\
\text { tals }\end{array}$ & $.00^{4}$ & $\begin{array}{r}0 \\
.00\end{array}$ & $\begin{array}{r}0 \\
.00\end{array}$ & 5000.00 & 120.00 & 28.00 \\
\hline \multicolumn{2}{|c|}{ TOTAL } & $\begin{array}{r}8 \\
.00\end{array}$ & $\begin{array}{r}6 \\
.00\end{array}$ & $\begin{array}{r}6 \\
.00\end{array}$ & 25000.00 & 600.00 & 140.00 \\
\hline
\end{tabular}

Table $\mathrm{N}^{\circ} 3$ shows the horizontal elements metrics according to the sectorization carried out.

Table $\mathbf{N}^{\circ}$ 4. Load balancing per sector unit

\begin{tabular}{|c|c|c|c|c|c|c|}
\hline Carga Scale & 8 & hrs & & & & \\
\hline Activity & $\begin{array}{c}\text { qua } \\
\text { ntity }\end{array}$ & Unt & Rend & Unt & $\mathrm{HH}$ & crew \\
\hline $\begin{array}{c}\text { Vertical Steel } \\
\text { Vertical }\end{array}$ & $\begin{array}{l}300 \\
0\end{array}$ & $\mathrm{~kg}$ & 0.030 & $\mathrm{hh} / \mathrm{kg}$ & 90 & 11.25 \\
\hline Formwork & 120 & $\mathrm{~m} 2$ & 0.850 & $\mathrm{hh} / \mathrm{m} 2$ & 102 & 12.75 \\
\hline $\begin{array}{c}\text { Vertical Concrete } \\
\text { Horizontal }\end{array}$ & 30 & $\mathrm{~m} 3$ & 1.000 & $\mathrm{hh} / \mathrm{m} 3$ & 30 & 3.75 \\
\hline $\begin{array}{l}\text { Formwork } \\
\text { Horizontal Steel }\end{array}$ & $\begin{array}{l}120 \\
500\end{array}$ & $\mathrm{~m} 2$ & 1.200 & $\mathrm{hh} / \mathrm{m} 2$ & 144 & 18 \\
\hline Horizontal & 0 & $\mathrm{~kg}$ & 0.050 & $\mathrm{hh} / \mathrm{kg}$ & 250 & 31.25 \\
\hline Concrete & 28 & $\mathrm{~m} 3$ & 1.000 & $\mathrm{hh} / \mathrm{m} 3$ & 28 & 3.5 \\
\hline Totals & & & & & 644 & 80.5 \\
\hline
\end{tabular}

Table $\mathrm{N}^{\circ} 04$ shows the yield that would be obtained for each sector, we will take one and with the support of the Unit Price Analyses (APUS) we will determine the yield that would be obtained for each sector. [8] 
Tabla $\mathbf{N}^{\circ}$ 5. General load balancing

\begin{tabular}{|c|c|c|c|c|c|c|}
\hline Balance de Carga & 8 & hrs & & & & \\
\hline Activity & $\begin{array}{l}\text { quant } \\
\text { ity }\end{array}$ & Unt & Rend & Unt & $\mathrm{HH}$ & crew \\
\hline Vertical Steel & 1500 & & & & & \\
\hline Vertical & $\begin{array}{l}0.00 \\
600.0\end{array}$ & $\mathrm{~kg}$ & 0.030 & $\mathrm{hh} / \mathrm{kg}$ & 450 & 56.25 \\
\hline Formwork & 0 & $\mathrm{~m} 2$ & 0.850 & $\mathrm{hh} / \mathrm{m} 2$ & 510 & 63.75 \\
\hline Vertical Concrete & 150.0 & & & & & \\
\hline Horizontal & $\begin{array}{l}0 \\
600.0\end{array}$ & $\mathrm{~m} 3$ & 1.000 & $\mathrm{hh} / \mathrm{m} 3$ & 150 & 18.75 \\
\hline Formwork & 0 & $\mathrm{~m} 2$ & 1.200 & $\mathrm{hh} / \mathrm{m} 2$ & 720 & 90 \\
\hline Horizontal Steel & 2500 & & & & & \\
\hline Horizontal & $\begin{array}{l}0.00 \\
140.0\end{array}$ & $\mathrm{~kg}$ & 0.050 & $\mathrm{hh} / \mathrm{kg}$ & 1250 & 156.25 \\
\hline Concrete & 0 & $\mathrm{~m} 3$ & 1.000 & $\mathrm{hh} / \mathrm{m} 3$ & 140 & 17.5 \\
\hline Totals & & & & & 3220 & 402.5 \\
\hline
\end{tabular}

Table 5 shows the load balancing for a sector and for the entire project. The load balancing should be according to the specific activity to be performed

Tabla $\mathbf{N}^{\circ}$ 6. LookAhead

\begin{tabular}{|c|c|c|c|c|c|c|}
\hline \multicolumn{7}{|c|}{ ACTIVITY SEQUENCE } \\
\hline Activity & D1 & D2 & D3 & D4 & D5 & D6 \\
\hline Vertical Steel & $\mathbf{X}$ & & & & & \\
\hline Vertical Formwork & & $\mathbf{X}$ & & & & \\
\hline Vertical Concrete & & & $\mathbf{X}$ & & & \\
\hline $\begin{array}{l}\text { Horizontal } \\
\text { Formwork }\end{array}$ & & & & $\mathbf{X}$ & & \\
\hline Horizontal Steel & & & & & $\mathbf{X}$ & \\
\hline Horizontal & & & & & & $\mathbf{x}$ \\
\hline
\end{tabular}

Once this has been done, the lookAhead is prepared, as shown in Table $\mathrm{N}^{\circ} 6$. The activities, necessary resources, definition of processes and detection of the restrictions of each activity that cannot yet be executed must be visualized. [9] All those involved in the work must participate in the planning, the objective is to determine the plan for future weeks in order to alert with the request for resources. By making the train of activities, the person in charge of the work will be able to know how much progress will be made each day. [10]

know how much progress will be made each day, forecast exactly how much progress will be made on a given day, know how much will be spent per day on the work, have greater control of expenses on the work and advance the work with a minimum of rework. [11]

The delay of one item causes the delay of all items, delaying the system as a whole. [12]

Table

Table $\mathbf{N}^{\circ}$ 7. Project LookAhead

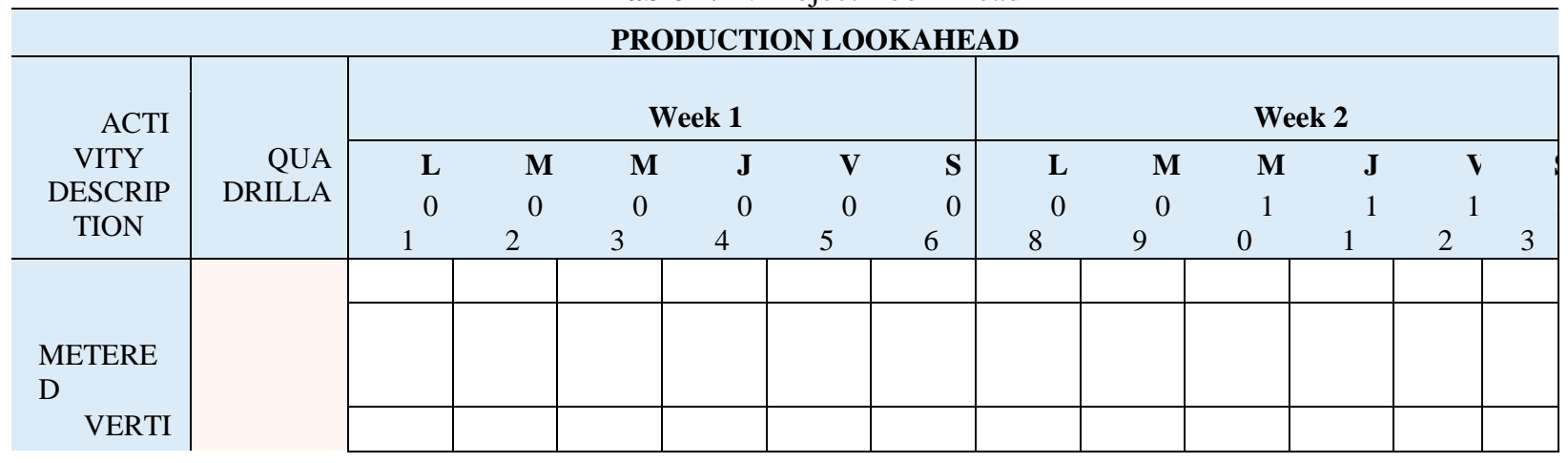




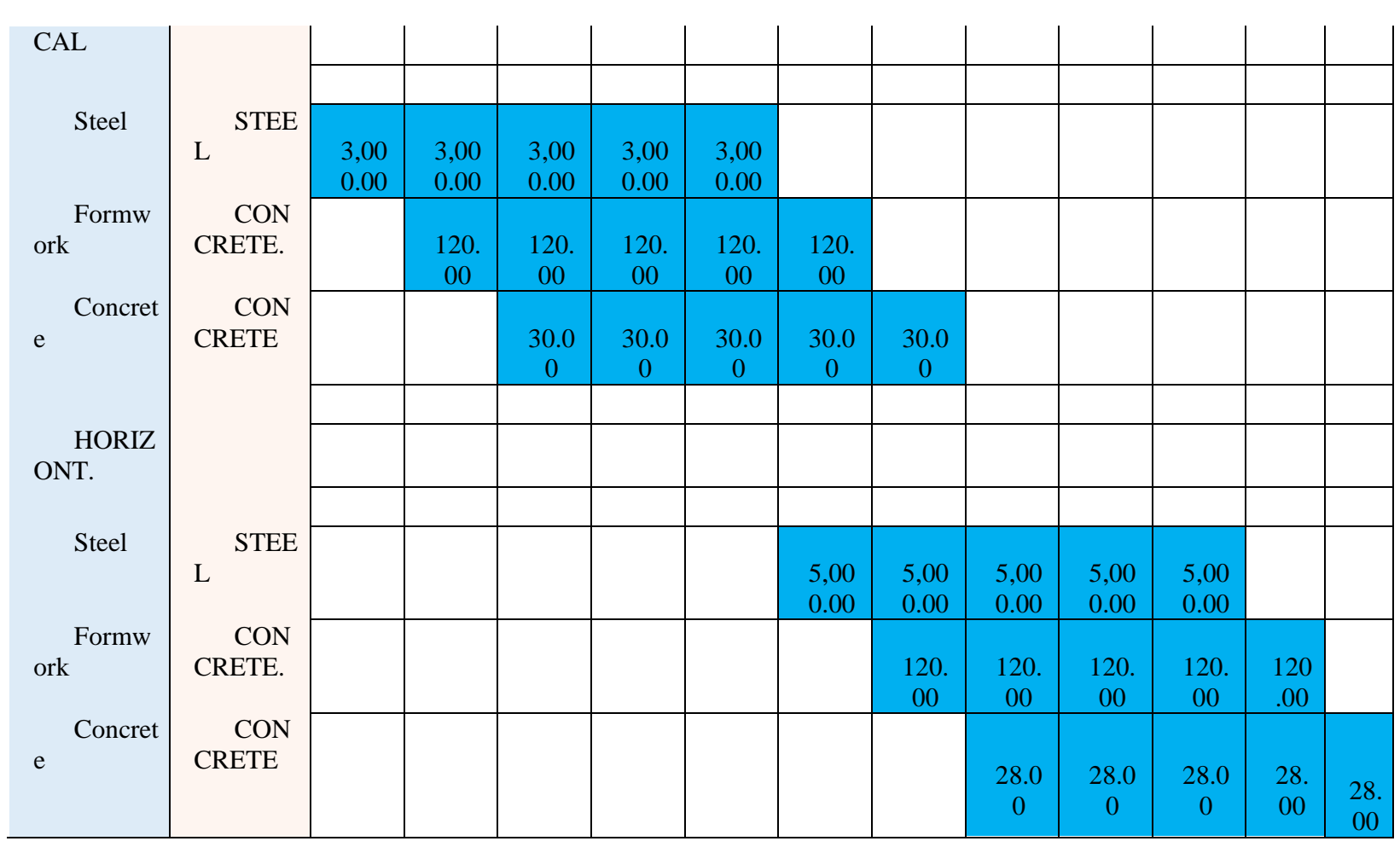

In Table $\mathrm{N}^{\circ} 7$, once the work sequence has been drawn up, we proceed to elaborate the sequence for activities balancing the loads of the project in question.

Table $\mathbf{N}^{\circ} 8$. Staffing Schedule

\begin{tabular}{|c|c|c|c|c|c|c|c|c|c|c|c|c|c|}
\hline & \multirow[b]{2}{*}{ ITEM } & \multicolumn{6}{|c|}{ Week 1} & \multicolumn{6}{|c|}{ Week 2} \\
\hline & & $\mathbf{L}$ & $\mathbf{M}$ & M & $\mathbf{J}$ & $\mathbf{V}$ & $\mathbf{S}$ & $\mathbf{L}$ & M & N & $\mathbf{J}$ & $\mathbf{V}$ & $\mathrm{s}$ \\
\hline EFFICI & & 0 & 0 & 0 & $\mathbf{0}$ & $\mathbf{0}$ & 0 & 0 & O & 1 & 1 & 1 & 1 \\
\hline ENCY & & 1 & 2 & 3 & 4 & 5 & 6 & 8 & 9 & 0 & 1 & 2 & 3 \\
\hline \multirow{5}{*}{$\begin{array}{l}\text { METRA } \\
\text { DO }\end{array}$} & STEEL & $\begin{array}{r}3 \\
\end{array}$ & 3 & 3 & 3 & 3 & 5 & 5 & 5 & 5 & 5 & 0 & $c$ \\
\hline & FOUNDA & & 1 & 1 & 1 & 1 & 1 & 1 & 1 & 1 & 1 & 1 & \\
\hline & TION & 0 & 20 & 20 & 20 & 20 & 20 & 20 & 20 & 20 & 20 & 20 & \\
\hline & CONCRE & & & 3 & 3 & 3 & 3 & 3 & 2 & 2 & 2 & 2 & 2 \\
\hline & TE & 0 & 0 & 0.0 & 0.0 & 0.0 & 0.0 & 0.0 & 8.0 & 8.0 & 8.0 & 8.0 & 8.0 \\
\hline EFFEC & STEEL & 1 & 1 & 1 & 1 & 1 & 3 & 3 & 3 & 3 & 3 & 0 & 0 \\
\hline TIVE & & 1.25 & 1.25 & 1.25 & 1.25 & 1.25 & 1.25 & 1.25 & 1.25 & 1.25 & 1.25 & .00 & .00 \\
\hline PERSONN & FOUNDA & 0 & 1 & 1 & 1 & 1 & 1 & 1 & 1 & 1 & 1 & 1 & c \\
\hline \multirow[t]{5}{*}{ EL } & TION & .00 & 2.75 & 2.75 & 2.75 & 2.75 & 2.75 & 8.00 & 8.00 & 8.00 & 8.00 & 8.00 & .00 \\
\hline & CONCRE & 0 & 0 & 3 & 3 & 3 & 3 & 3 & 3 & 3 & 3 & 3 & 3 \\
\hline & $\mathrm{TE}$ & .00 & .00 & .75 & .75 & .75 & .75 & .75 & .50 & .50 & .50 & .50 & .50 \\
\hline & TOTAL & 1 & 2 & 2 & 2 & 2 & 4 & 5 & 5 & 5 & 5 & 2 & 3 \\
\hline & & 1.25 & 4.00 & 7.75 & 7.75 & 7.75 & 7.75 & 3.00 & 2.75 & 2.75 & 2.75 & 1.50 & .50 \\
\hline \multirow[t]{7}{*}{ STAFF } & STEEL & 1 & 1 & 1 & 1 & 1 & 3 & 3 & 3 & 3 & 3 & 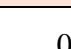 & 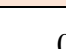 \\
\hline & & 1 & 1 & 1 & 1 & 1 & 1 & 1 & 1 & 1 & 1 & 0 & $\mathrm{c}$ \\
\hline & FOUNDA & 0 & 1 & 1 & 1 & 1 & 1 & 1 & 1 & 1 & 1 & 1 & 0 \\
\hline & TION & 0 & 3 & 3 & 3 & 3 & 3 & 8 & 8 & 8 & 8 & 8 & c \\
\hline & $\begin{array}{r}\text { CONCRE } \\
\text { TE }\end{array}$ & 0 & 0 & 4 & 4 & 4 & 4 & 4 & 4 & 4 & 4 & 4 & 4 \\
\hline & TOTAL & 1 & 2 & 2 & 2 & 2 & 4 & 5 & 5 & 5 & 5 & 2 & \\
\hline & & 1.00 & 4.00 & 8.00 & 8.00 & 8.00 & 8.00 & 3.00 & 3.00 & 3.00 & 3.00 & 2.00 & .00 \\
\hline
\end{tabular}


In table $\mathrm{N}^{\circ}$, with the elaboration of the look ahead, according to the amount of meter to be performed, we proceed with the personnel schedule to maintain a correct rhythm of work without having to dismiss and then hire by days. [13]

Table No9. Restriction analysis

\begin{tabular}{|c|c|c|c|c|c|c|c|c|c|c|c|c|c|c|c|c|c|c|c|}
\hline \multicolumn{6}{|c|}{ Restriction analysis } & \multicolumn{7}{|c|}{ WEEK 1} & \multicolumn{7}{|c|}{ WEEK 2} \\
\hline $\begin{array}{l}\text { I } \\
\text { TE } \\
\text { M }\end{array}$ & $\begin{array}{c}\text { W } \\
\text { HER } \\
\text { E IT } \\
\text { COM } \\
\text { ES } \\
\text { FRO } \\
\text { M }\end{array}$ & $\begin{array}{c}\text { DESC } \\
\text { RIPTION } \\
\text { OF THE } \\
\text { ACTIVIT } \\
\text { Y }\end{array}$ & $\begin{array}{c}\text { DESC } \\
\text { RIPTION } \\
\text { OF THE } \\
\text { RESTRIC } \\
\text { TION }\end{array}$ & $\begin{array}{c}\text { DT } \\
\mathbf{E}\end{array}$ & $\begin{array}{l}\text { RESPO } \\
\text { NSABLE }\end{array}$ & $\bar{\theta}$ & ชิ & $\dddot{8}$ & $\Xi$ & $\mathscr{8}$ & ๕ & s & $\stackrel{8}{\circ}$ & 8 & 으 & $=$ & $\mathcal{Z}$ & $\dddot{2}$ & \pm \\
\hline .01 & & $\begin{array}{l}\text { VERTI } \\
\text { CAL }\end{array}$ & & & & & & & & & & & & & & & & & \\
\hline $\begin{array}{l}1 \\
.01 . \\
01\end{array}$ & $\mathrm{C}^{\mathrm{O}}$ & Steel & $\begin{array}{l}\text { Arrival } \\
\text { of } \\
25,000.00 \\
\mathrm{~kg} \text { of steel } \\
\text { at the site }\end{array}$ & $1^{0}$ & GP. & $x$ & & & & & & & & & & & & & \\
\hline $\begin{array}{l}1 \\
.01 . \\
02\end{array}$ & $\begin{array}{c}\mathrm{C} \\
\text { ontrac } \\
t\end{array}$ & & $\begin{array}{l}\text { Arrival } \\
\text { of } 11 \text { Steel } \\
\text { Operators }\end{array}$ & $1^{0}$ & ADM. & $x$ & & & & & & & & & & & & & \\
\hline $\begin{array}{l}1 \\
.01 . \\
03\end{array}$ & $\begin{array}{c}\text { C } \\
\text { ontrac } \\
t\end{array}$ & & $\begin{array}{l}\text { Safety } \\
\text { talk to the } \\
11 \\
\text { operators }\end{array}$ & $1^{0}$ & PDR & $x$ & & & & & & & & & & & & & \\
\hline $\begin{array}{l}1 \\
.01 . \\
04\end{array}$ & $\mathrm{~S}^{\mathrm{O} /}$ & $\begin{array}{l}\text { Formw } \\
\text { ork }\end{array}$ & $\begin{array}{l}\text { Arrival } \\
\text { of } 120 \mathrm{~m} 2 \\
\text { of } \\
\text { formwork }\end{array}$ & $2^{0}$ & JOT. & & $\mathbf{x}$ & & & & & & & & & & & & \\
\hline $\begin{array}{l}1 \\
.01 . \\
05\end{array}$ & $\begin{array}{c}\mathrm{C} \\
\text { ontrac } \\
t\end{array}$ & & $\begin{array}{l}\text { Arrival } \\
\text { of } 13 \\
\text { carpentry } \\
\text { operators }\end{array}$ & $2^{0}$ & ADM. & & $\mathbf{x}$ & & & & & & & & & & & & \\
\hline $\begin{array}{l}1 \\
.01 . \\
06\end{array}$ & $\begin{array}{c}\mathrm{C} \\
\text { ontrac } \\
\mathrm{t}\end{array}$ & & $\begin{array}{l}\text { Safety } \\
\text { talk to the } \\
13 \\
\text { operators }\end{array}$ & $2^{0}$ & PDR & & $\mathbf{x}$ & & & & & & & & & & & & \\
\hline $\begin{array}{l}1 \\
.01 . \\
08\end{array}$ & $\begin{array}{c}\mathrm{C} \\
\text { ontrac } \\
t\end{array}$ & $\mathrm{e}^{\text {Concret }}$ & $\begin{array}{l}\text { Arrival } \\
\text { of } 4 \\
\text { concrete } \\
\text { workers } \\
\end{array}$ & $3^{0}$ & ADM. & & & $\mathbf{x}$ & & & & & & & & & & & \\
\hline $\begin{array}{l}1 \\
.01 . \\
09\end{array}$ & $\begin{array}{c}\mathrm{C} \\
\text { ontrac } \\
t\end{array}$ & & \begin{tabular}{l}
\multicolumn{1}{c}{ Safety } \\
talk to the 4 \\
concrete \\
workers
\end{tabular} & $3^{0}$ & PDR & & & $\mathbf{x}$ & & & & & & & & & & & \\
\hline $\begin{array}{c}1 \\
.01 . \\
10\end{array}$ & $\mathrm{C}^{\mathrm{O} /}$ & e Concret & $\begin{array}{l}\text { Arrival } \\
\text { of the } \\
30 \mathrm{~m} 3 \text { of } \\
\text { ready- } \\
\text { mixed } \\
\text { concrete }\end{array}$ & $4^{0}$ & JOT. & & & & $\mathbf{x}$ & $\mathbf{x}$ & $\mathbf{x}$ & & & & & & & & \\
\hline $\begin{array}{l}1 \\
.01 . \\
13\end{array}$ & $\begin{array}{l}\mathrm{O} / \\
\mathrm{C}\end{array}$ & e & $\begin{array}{l}\text { Arrival } \\
\text { of } 30 \mathrm{~m} 3 \text { of } \\
\text { ready- } \\
\text { mixed } \\
\text { concrete }\end{array}$ & $8^{0}$ & JOT. & & & & & & & & $\mathbf{x}$ & & & & & & \\
\hline .02 & & $\begin{array}{l}\text { HORIZ } \\
\text { ONTAL }\end{array}$ & & & & & & & & & & & & & & & & & \\
\hline $\begin{array}{l}1 \\
.02 . \\
01\end{array}$ & $\mathrm{C}^{\mathrm{O} /}$ & Steel & $\begin{array}{l}\text { Arrival } \\
\text { of } 15,000 \\
\mathrm{~kg} \text { of steel } \\
\text { at site }\end{array}$ & $8^{0}$ & JA. & & & & & & & & $\mathbf{x}$ & & & & & & \\
\hline
\end{tabular}




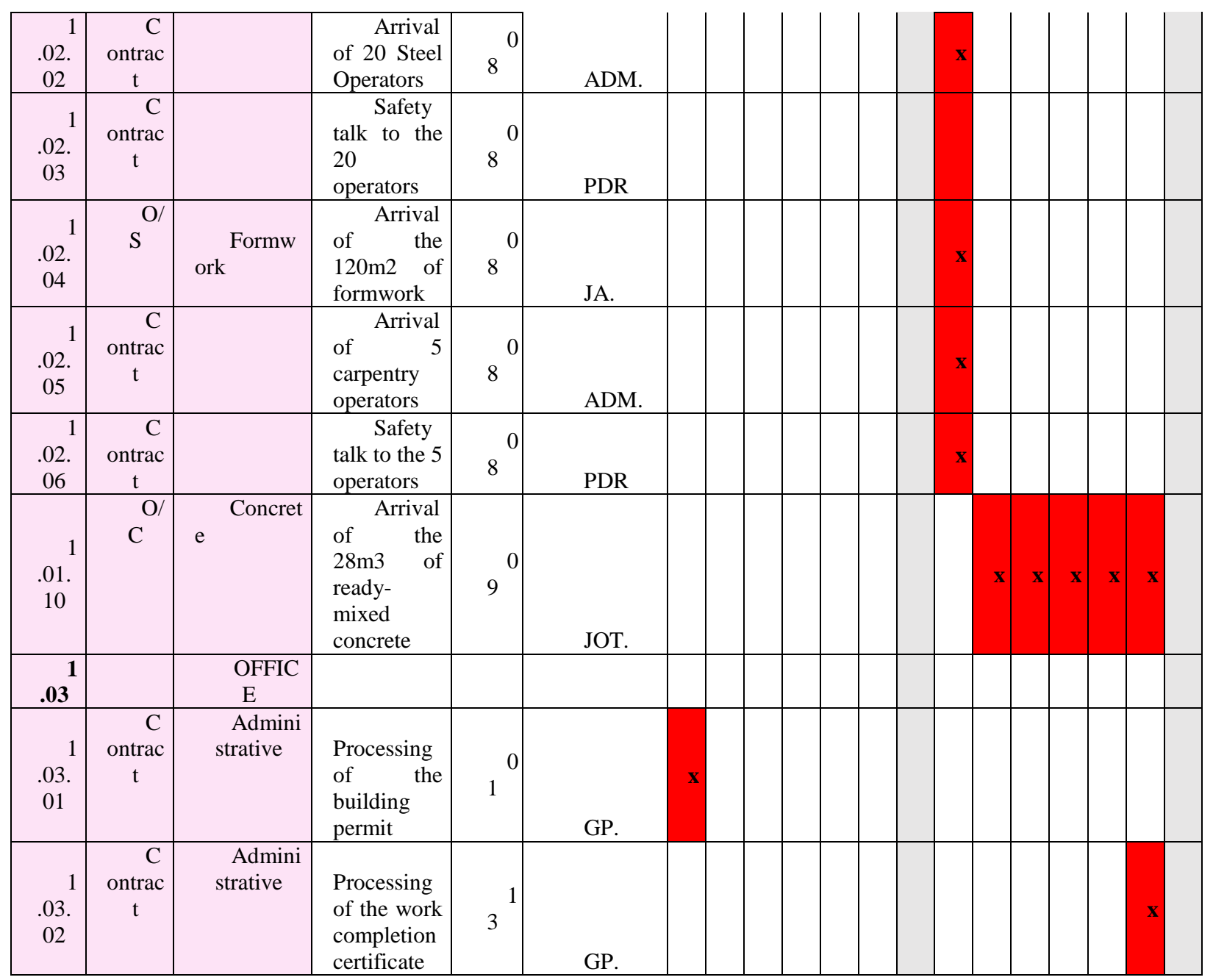

In Table $\mathrm{N}^{\circ}$ 9, once this is concluded, we proceed with the analysis of restrictions to avoid setbacks during the execution of the work.

\section{Results}

Table $\mathrm{N}^{\circ} 10$. performance

by sector

performance

\begin{tabular}{|c|c|c|c|c|}
\hline Activity & $\begin{array}{l}\text { Quadril } \\
\text { la } \\
\end{array}$ & HH & Table & $\mathrm{N}^{\circ} 11$ \\
\hline Vertical Steel & 56.00 & $\begin{array}{l}448 . \\
00\end{array}$ & & \\
\hline Vertical & & 512. & & \\
\hline Formwork & 64.00 & 00 & & \\
\hline $\begin{array}{l}\text { Vertical } \\
\text { Concrete } \\
\text { Horizontal }\end{array}$ & 19.00 & $\begin{array}{l}152 . \\
00 \\
720 .\end{array}$ & & \\
\hline Formwork & 90.00 & 00 & & \\
\hline $\begin{array}{l}\text { Horizontal } \\
\text { Steel }\end{array}$ & 156.00 & $\begin{array}{l}124 \\
8.00\end{array}$ & & \\
\hline Horizontal & & 144. & & \\
\hline Concrete & 18.00 & 00 & & \\
\hline Totals & 403.00 & $\begin{array}{l}322 \\
4.00\end{array}$ & & \\
\hline
\end{tabular}




\begin{tabular}{ccc}
\hline Activity & $\begin{array}{c}\text { Quadri } \\
\text { lla }\end{array}$ & $\begin{array}{c}\text { H } \\
\text { H }\end{array}$ \\
\hline \hline Vertical Steel & & 88. \\
& 11.00 & 00 \\
Vertical & & 10 \\
Formwork & 13.00 & 4.00 \\
Vertical & & 24. \\
Concrete & 3.00 & 00 \\
Horizontal & & 14 \\
Formwork & 18.00 & 4.00 \\
Horizontal & & 24 \\
Steel & 31.00 & 8.00 \\
Horizontal & & 32. \\
Concrete & 4.00 & 00 \\
\hline \hline Totals & & 64 \\
& 80.00 & 0.00 \\
& & \\
Efficiency & & $\mathbf{9 9}$ \\
\hline
\end{tabular}

Table $\mathrm{N}^{\circ} 10$ and $\mathrm{N}^{\circ} 11$ show that an efficiency of $100 \%$ will be obtained by using the calculated crew in accordance with the yields. [14]

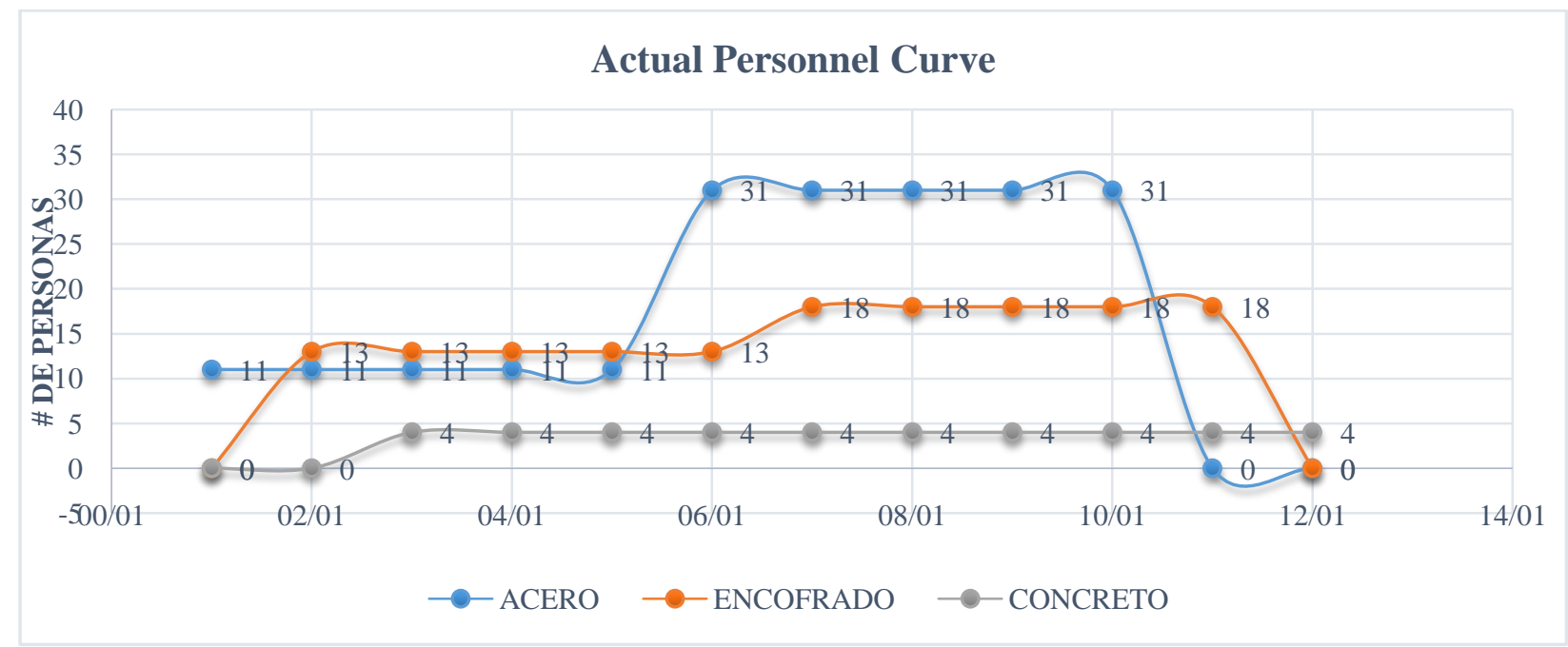

Figure 6. performance by sector

Figure 6, after performing the lookAhead, shows the actual personnel curve according to the distribution in time and deadlines in order to meet the needs: manpower, equipment, consumables, critical materials, subcontracting and others. [15]

Tabla $\mathbf{N}^{\circ} 12$. performance by sector

\begin{tabular}{ccc}
\hline \multicolumn{3}{c}{ CONSTRAINT ANALYSIS } \\
\hline & WEEK 1 & WEEK 2 \\
\hline \hline TOTAL NO. OF RESTRICTIONS & 12 & 13 \\
\% OF RESTRICTIONS PER WEEK & $48 \%$ & $52 \%$ \\
\hline
\end{tabular}

After analyzing the restrictions, we can see that during the execution of the work, during the first week there will be a total of $48 \%$ of the restrictions and for the second week there will be $52 \%$.

\section{Conclusions}

In order to maintain a correct personnel curve, it is necessary to try to balance the curves so as not to have paralyzed personnel during the execution of the work.It is possible to try to reduce the sectorization in order to minimize the work areas, but it would increase the workloads causing the increase of crews. 
The sectorization helps to optimize the production of workers through repetitive work, also helps to have greater control over the items allowing to reduce the time of the process.Perform the Loos Ahead in order to create a "shield" 3 to 5 weeks in advance.

This allows us to foresee what is needed so that future activities can be carried out.For the constraint analysis it should be done formally (meeting and minutes of agreements), concrete action should be taken on the identified constraints, in addition to always assign responsible for each constraint, set deadlines and follow $\mathrm{u}$

\section{References}

Lung, L., Shaurette, M. (2018), "El trabajo colaborativo, aplicado al diseño y la construcción, para promover la construcción de edificios verdes en Perú", Rev. ing. constr. Vol.33 No.2, pp.1. Recuperado de: http://dx.doi.org/10.4067/S0718-50732018000200183

Hinostroza, D. Manosalva, O. (2015). aplicación de last planner en edificaciones multifamiliares. Lima, Perú. [Tesis de grado, Universidad Ricardo Palma]. Recuperado de: https://repositorio.urp.edu.pe/bitstream/handle/URP/2224/manosalva_oo-

Buleje, K (2012). Productividad en la construcción de un condominio aplicando conceptos de la filosofía lean construcción. Lima, Perú. [Tesis de grado, Universidad Católica del Perú]. Recuperado de: http://tesis.pucp.edu.pe/repositorio/bitstream/handle/20.500.12404/1691/BULEJE_KENNY_CONDOMINIO _LEAN_CONSTRUCTION.pdf?sequence $=1 \&$ isAllowed $=\mathrm{y}$

Chokewanca, V. Sotomayor, J. (2018). Sistema last planner para mejorar la planificación en la obra civil del centro de salud picota - san martín. Lima, Perú. [Tesis de grado, Universidad San Martin de Porres]. Recuperado

de: http://repositorio.usmp.edu.pe/bitstream/handle/20.500.12727/4235/chokewanka_sotomayor.pdf?sequence=3

Andrade, M., Arrieta, B. (2011), "Last planner system results in subcontract construction company", Revista de construcción. Vol.10 No.1, pp.36-52. Recuperado de: http://dx.doi.org/10.4067/S0718-915X2011000100005

Cornejo, K. Gonzales, F. Tapia, V. (2017). Implementación de last planner system en actividades de concreto armado para proyectos de edificacion industrial. Lima, Perú. [Tesis de maestria, Universidad Peruana de Ciencias Aplicadas]. Recuperado de: https://repositorioacademico.upc.edu.pe/bitstream/handle/10757/623900/Cornejo_lk.pdf?sequence=13

Angeli, C. (2017). Implementación del sistema last planner en edificación en altura en una empresa constructora: estudio de casos de dos edificios en las comunas de Las Condes y San Miguel. Santiago, Chile. [Tesis de grado, Universidad Andres Bello]. Recuperado de: http://repositorio.unab.cl/xmlui/bitstream/handle/ria/4601/a120179_Angeli_C_Implementacion_del_sistema_ last_planner_tesis_2017.pdf?sequence $=1 \&$ isAllowed=y

Mendoza, W. (2019). implementación del last planner y la metodología del valor ganado en proyectos civiles "construccion de puentes", red vial 5- huacho. Huancayo, Perú. [Tesis de grado, Universidad Nacional del Centro del Perú]. Recuperado de: http://repositorio.uncp.edu.pe/bitstream/handle/UNCP/5555/T010_44573636_T.pdf?sequence=1\&isAllowed $=\mathrm{y}$

Jauregui, C. Pairazaman, C. (2016). Aplicación del sistema last planner de la filosofía lean construction para la generacion de valor en la obra conjunto residencial golf los andes - etapa II, lurigancho - chosica 2014. Lima, Perú. [Tesis de grado, Universidad Privada Antenor Orrego]. Recuperado de: http://repositorio.upao.edu.pe/bitstream/upaorep/2057/1/RE_ING.CIVIL_CESAR.JAUREGUI_JORGE.PAI RAZAMAN_EQUIPO.DE.PROYECTO.GENERACION.DE.VALOR_DATOS.pdf

Hinostroza, D. Manosalva, O. (2015). Aplicación de last planner en edificaciones multifamiliares. Lima, Perú. [Tesis de grado, Universidad Ricardo Palma]. Recuperado de: http://repositorio.urp.edu.pe/bitstream/handle/URP/2224/manosalva_oo-

Gonzales, A. (2012). Propuesta de implementación del sistema last planner con el apoyo de modelación 4d para la obra gruesa de edificaciones. Santiago, Chile. [Tesis de grado, Universidad de Chile]. Recuperado

Carranza, R. Tejada, C. (2018). Estudio comparativo de la implementación del last planner system y el sistema tradicional en la construcción de una tienda comercial makro supermayorista, Comas - Lima. Lima, Perú. [Tesis de grado, Universidad San Pedro]. Recuperado de: http://repositorio.usanpedro.edu.pe/bitstream/handle/USANPEDRO/5481/Tesis_58046.pdf?sequence=1\&isA llowed=y

Tucto, G. Sanchez, V. (2017). Metodología de aplicación de la filosofía lean construction y last planner system en la región san martín. Lima, Perú. [Tesis de grado, Universidad Nacional de San Martin - Tarapoto]. Recuperado

de: http://repositorio.unsm.edu.pe/bitstream/handle/11458/2589/CIVIL\%20\%20Gladis\%20Karol\%20Tucto\%20P inedo.pdf? sequence $=1 \&$ is Allowed $=y$ 
Herrera, R., Muñoz, F., Avila, B (2020), "Principales requerimientos de una herramienta TI basada en last planner® system”, Rev. ing. constr. Vol.35 No.2, pp.1. Recuperado de: http://dx.doi.org/10.4067/S071850732020000200126

Diaz, L., De olivera, M., Pucharelli, P., Pinzón, J. (2019), “Integración entre el sistema last planner y el sistema de gestión de calidad aplicados en el sector de la construcción civil", Rev. ing. constr. Vol.34 No.2, pp.1. Recuperado de: http://dx.doi.org/10.4067/S0718-50732019000200146 